\title{
EVALUATING ANTI-HYPERTENSION MEDICATIONS USAGE ON PATIENT CARE ONLINE BLOGS
}

\author{
Nil Shah, Jinan Fiaidhi and Sabah Mohammed \\ Department of Computer Science, Lakehead University, ON, Canada \\ \{nshah, jfiaidhi, mohammed\}@lakeheadu.ca
}

\begin{abstract}
Throughout history, the area of drug discovery and development has been a financial strain due to the associated high costs. In order to offset this financial burden, drug companies are continually increasing the price of medications to consumers. Some consumers remain unaware of the types of medications available on the market and the gap in cost between these types. The two main types of medications available on the market are: 1) Generic drugs and 2) Brand name drugs. The purpose of this paper is to examine the similarities and difference of generic and brand name drugs from patient's reviews at major medications blogs like dugs.com. A subjectivity sentimental analysis framework has been developed that can effectively score these reviews without going into the complexities of using natural language or machine learning approaches. The developed framework use a well-known rule based subjectivity API known as VADER besides an effective web crawler. Results of this analysis shows more sentiments are with the generic antihypertension drugs compared to the brand drugs. The validation of these results was based on Google Trends. More concrete analysis of the results on wider list of medications as well as more blogs is left to our future work.
\end{abstract}

Keywords - Sentimental analysis, Antihypertension drugs, Branded drugs, Generic drugs, Web crawling, Text analytics

\section{INTRODUCTION}

Recent trends regarding causes of death and age-adjusted incidence of major cardiovascular events in the United States have shown an impressive decline in the risk of myocardial infarction and stroke [1] which have been attributed to better preventive therapies, including the use of drugs to lower blood pressure (aka hypertension) [2]. However, many antihypertensive drugs are now available in generic formulations at fractions of the cost of their branded counterparts. According to the FDA regulations, a generic drug product is one that is comparable to an innovator drug product in dosage form, strength, route, quality, performance characteristics and intended use. The FDA rules are tagged as "abbreviated" because they do not require going through clinical trial data in order to establish safety and efficacy. The only requirement is to show that the generic drug is bioequivalent to the brand drug. The test for such equivalence may include simple laboratory tests like maintaining the same plasma concentration as the brand drug in healthy volunteers. This FDA requirement caused many clinical and pharmaceutical researches to attempt comparing Brand vs Generic drugs. However, this

Received: January 3, 2019

Reviewed: March 21, 2019

Accepted: March 27, 2019

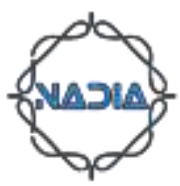


comparison has been done without involving the drug consumer's opinions on their usage (branded vs generic). Compared to other industries, pharmaceutical companies are arriving late to the Big Data and Social Media analytics. Historically, pharmaceutical companies have seen tremendous profit margins and thus there were in a position not to take on expansive and technical informatics initiatives to drive their commercial focus. Now, with the healthcare reform and new regulations, the whole picture has been changed. These companies are currently implementing Big Data Analytics strategies for a number of initiatives, including customer targeting, assessing performance, characterizing disease states and patient populations. Actually the ability to access, analyze, and manage vast volumes of data while rapidly evolving the Information Architecture may help to improve the efficiency of the drug discovery, marking and development process. In this direction we need to note that Big Data is only helpful when it's the right data, and that can be a struggle for a whole host of reasons when it comes to the issue of comparing the consumer's opinions on hypertension drugs. Analyzing generic data streams on hypertension drugs will be meaningless without distinguishing between single agent hypertension drug class (e.g., diuretics, beta-blockers, calcium antagonists, ACE inhibitors and ARBs) and multi-agent hypertension drug class (e.g., includes two- or more drug antihypertensive drug combination products). Harnessing pharmaceutical big data (aka Big Pharma), and distilling it into relevant insights requires correlating it to the right products to make the information valuable and actionable. This research paper is an attempt to explore using the Big Data analytics in providing insights on the difference between generic and branded antihypertension drugs from patients and consumers point of view through analyzing big data collected from specialized social media blogs like drugs.com ${ }^{1}$, webmed ${ }^{2}$ and patientslikeme ${ }^{3}$ where detailed drug usage reviews are provided.

\section{SENTIMENT ANALYSIS FOR MEDICATIONS COMPARISON}

Researchers have used various approaches for sentiment analysis. Most of the early studies were focused on document-level analysis for assigning the sentiment orientation of a document or a review [3]. However, these document-level sentiment analysis approaches are less effective when review texts are rather short or complex to be analyzed by simple natural language processing (NLP) and scoring techniques. Even the use of machine learning techniques that overcome the barriers of NLP techniques fails to correctly reflect the context of reviews sentiments as it treats reviews based on bag-fwords model. In this paper we have adopted an approach that computes sentiments of drugs reviews based on the subjectivity of the review text along with the average rating of the drug under review. Our developed approach avoids the difficulties associated with both the NLP approach as well as the machine learning approach. Figure 1 illustrates how reviewers comment and score a drug like Amlodipine. For the purpose of this study we have used twenty two drugs for treating hypertension with the help of a local pharmacist. Table I illustrates the selected drugs and their classification as generic and brand drugs.

\footnotetext{
${ }^{1}$ https://www.drugs.com/

${ }^{2}$ https://www.webmd.com/

${ }^{3} \mathrm{https}: / /$ www.patientslikeme.com/
} 
Table I. Antihypertension drug sample.

$\begin{array}{llc}\text { Brand name } & \text { Generic name } & \text { Generic avalilable in Canada } \\ \text { Tenormin } & \text { atenolol } & \mathrm{Y} \\ \text { Norvasc } & \text { amlodipine } & \mathrm{Y} \\ \text { Adalat XL } & \text { nifedipine } & \mathrm{Y} \\ \text { Altace } & \text { ramipril } & \mathrm{Y} \\ \text { Mavik } & \text { trandolapril } & \mathrm{N} \\ \text { Vasotec } & \text { enalapril } & \mathrm{Y} \\ \text { Coversyl } & \text { perindopril } & \mathrm{N} \\ \text { Diovan } & \text { valsartan } & \mathrm{Y} \\ \text { Atacand } & \text { candesartan } & \text { y } \\ \text { Mircardis } & \text { telmisartan } & \mathrm{Y} \\ \text { Cozaar } & \text { losartan } & \mathrm{Y}\end{array}$

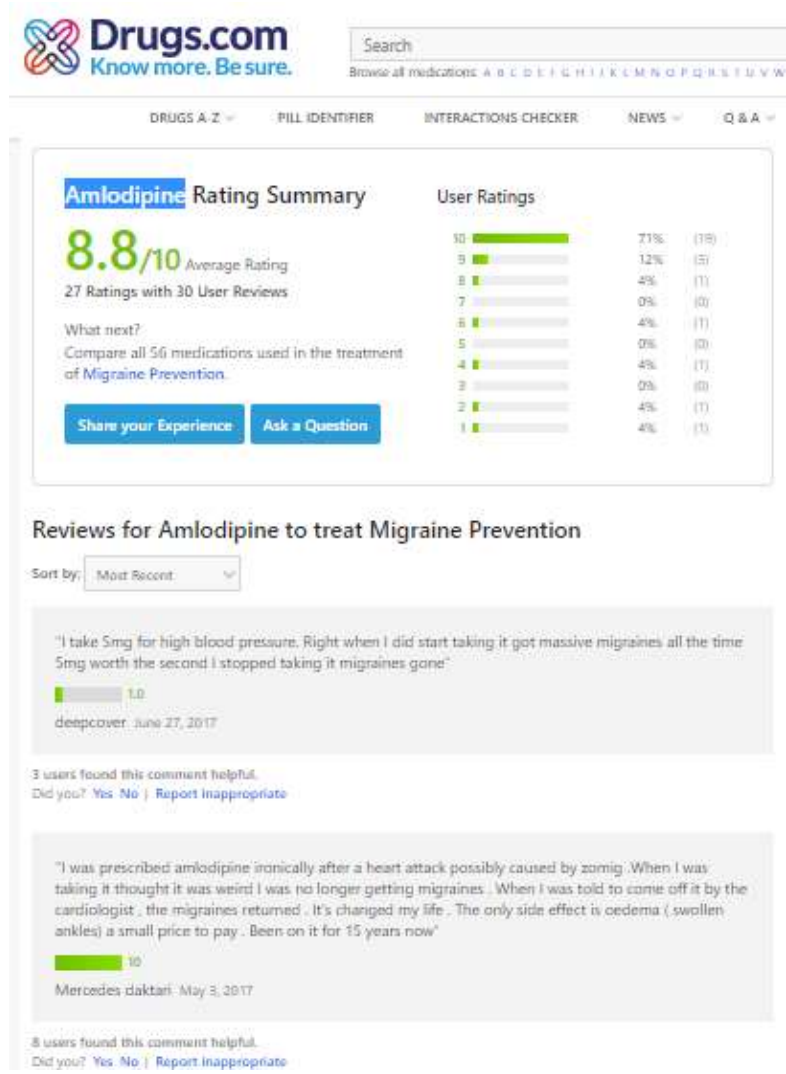

Fig. 1 A Sample of Reviews for Amlodipine

Our approach employs two major components: A drug targeting crawler and VADER $\mathrm{API}^{4}$ for scoring the subjectivity of the drugs reviews. VADER [4] is a simple rule-based model for general sentiment analysis, and it is a powerful approach that outperforms in eleven approaches notably other approaches used for sentiment scoring including LIWC, ANEW, the General Inquirer, SentiWordNet, and machine learning oriented techniques relying on Naive Bayes, Maximum Entropy, and Support Vector Machine (SVM). Figure 2 illustrate the workflow sequence of the processes used in our approach.

${ }^{4}$ https://github.com/cjhutto/vaderSentiment 


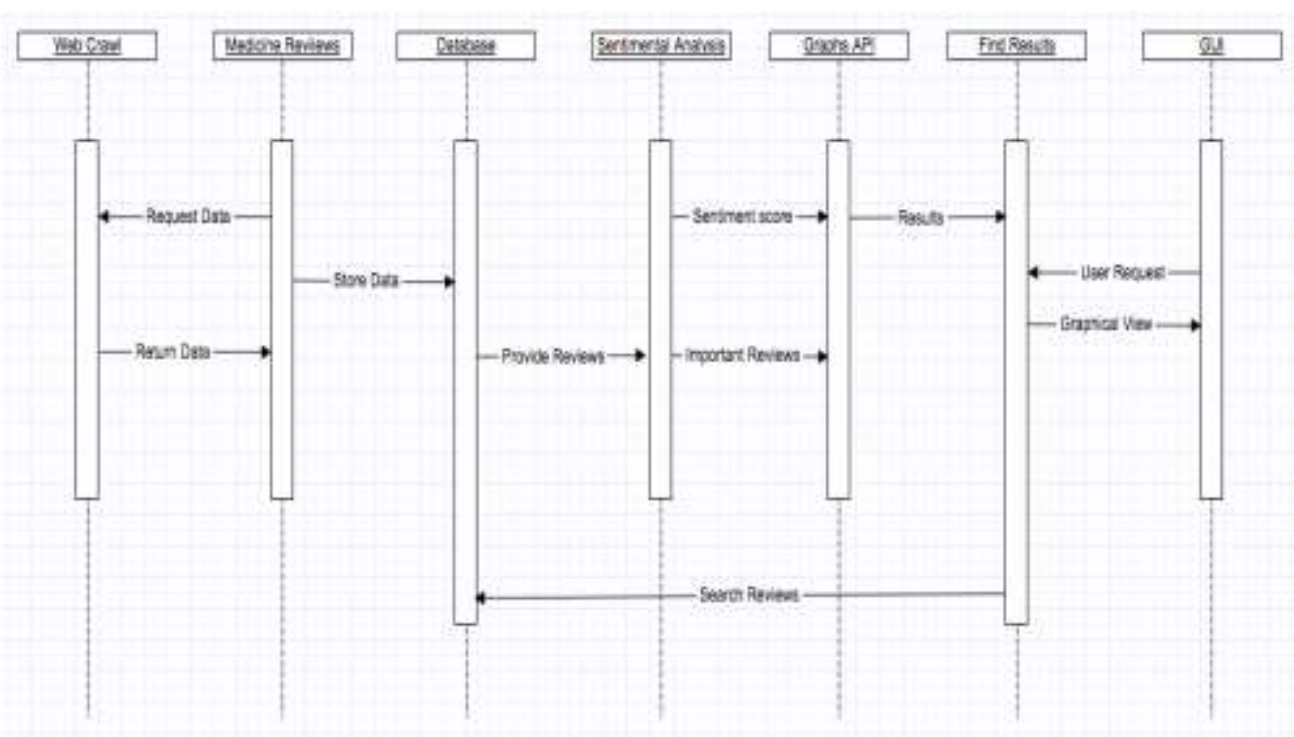

Fig. 2 The Subjectivity Scoring Workflow Sequence.

\subsection{CRAWLING DRUG REVIEWS USING SCRAPY}

Scrapy is a python application framework for crawling web sites and extracting structured data which can be used for a wide range of useful applications, like data mining, information processing or historical archival. Scrapy defines important methods like name(), start_requests() and parse(). The parse() method usually parses the response, extracting the scraped data as dicts and also finding new URLs to follow and creating new requests (Request) from them. Figure 3 illustrates how we can use scrapy to extract reviews from drugs.com.

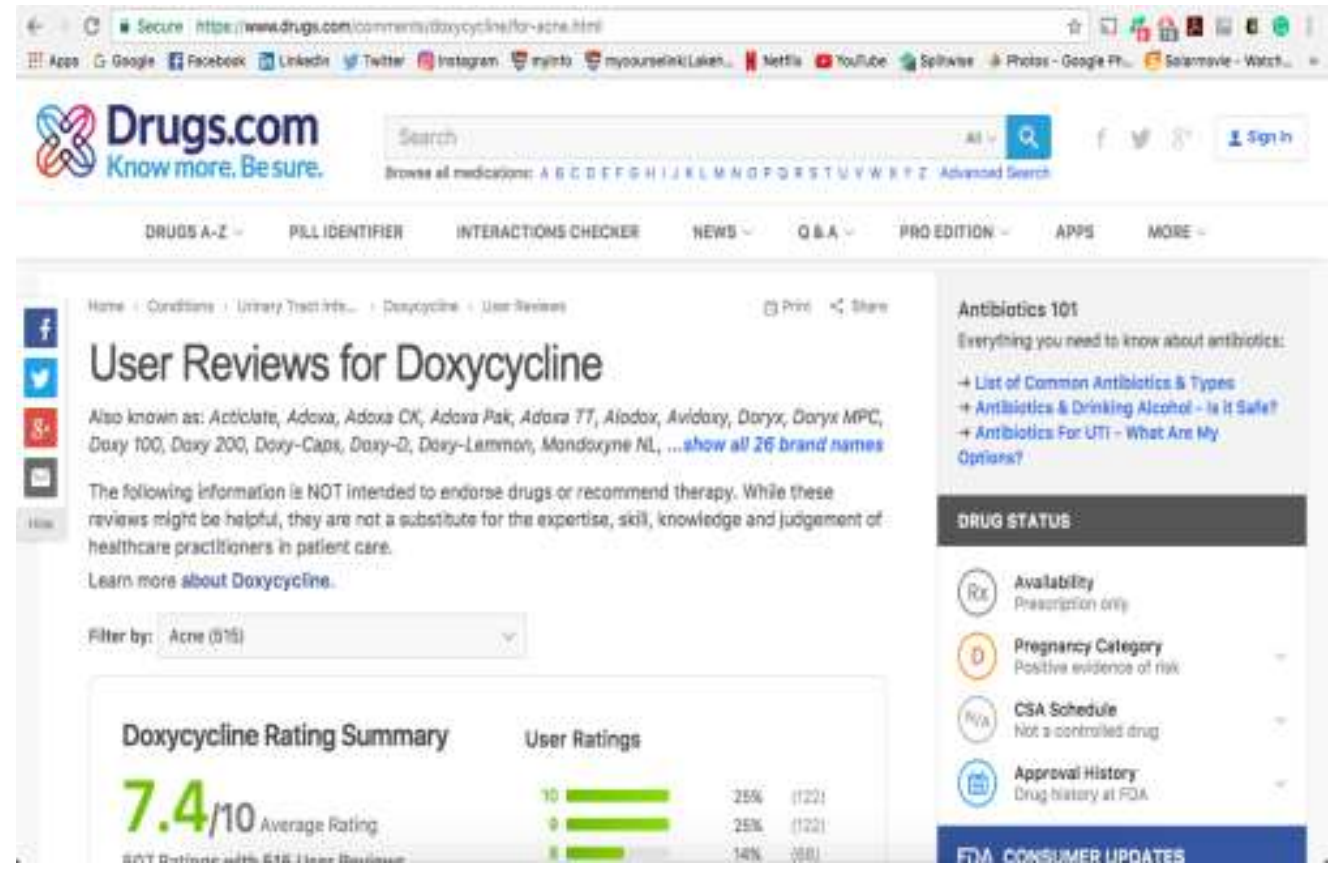




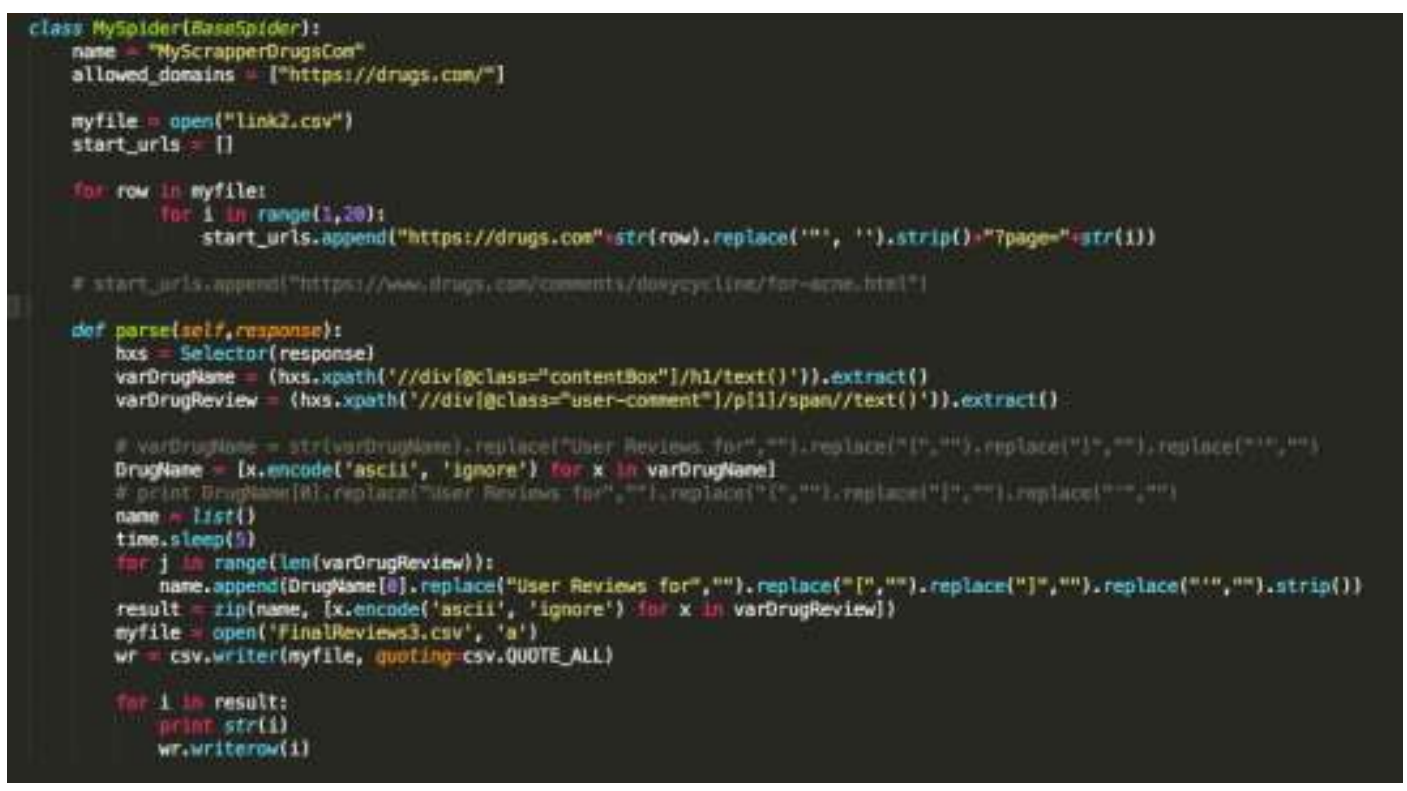

Fig. 3 Crawling Drugs Reviews using Scrapy for Drugs.Com

At the end, we can store the data in any format we want. Figure 4 illustrate the resulted data.

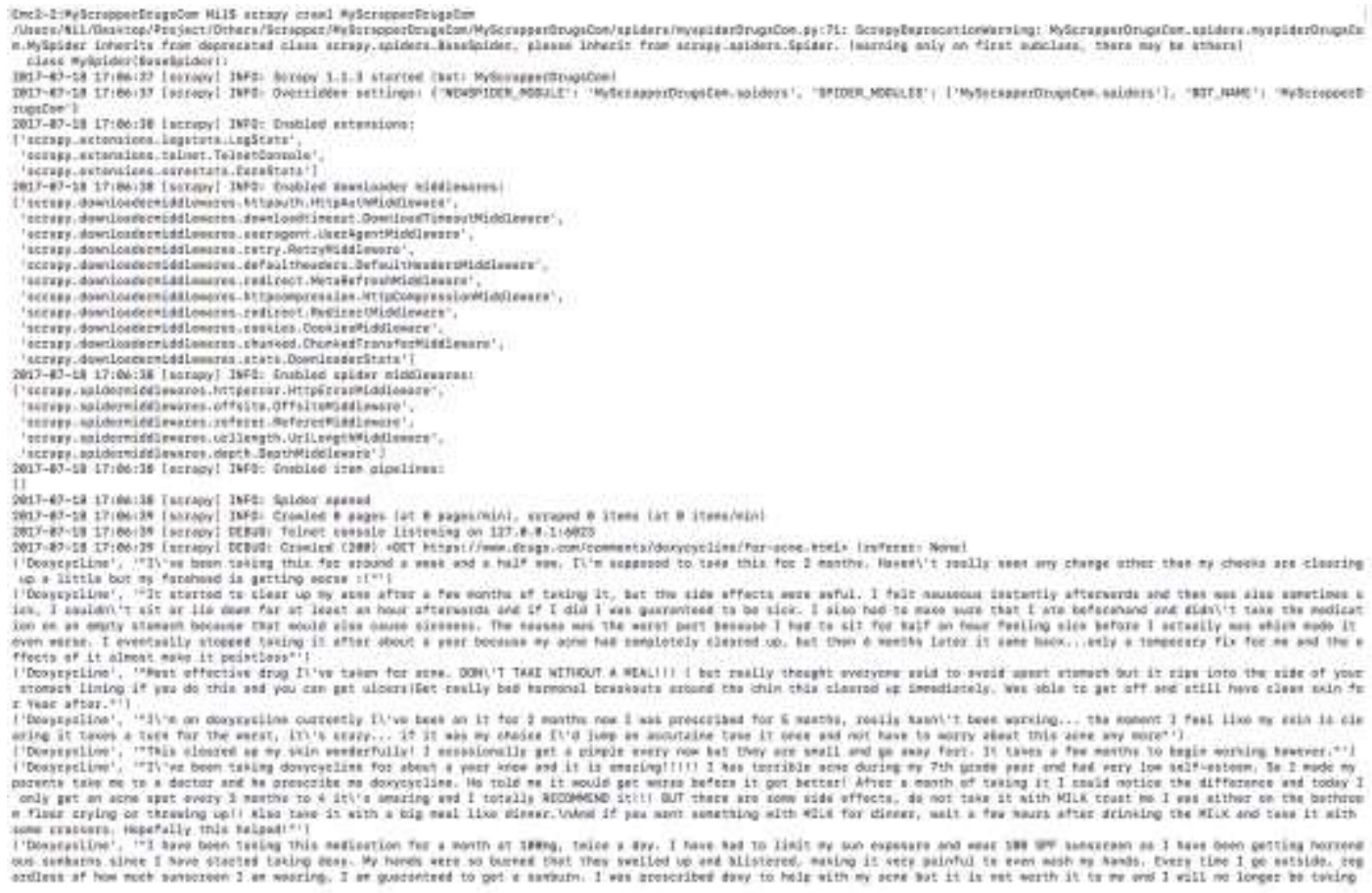

Fig. 4 Snapshot of the Resulted Data from Drugs.com.

We used for visualizing the drugs sentiment data the Django framework ${ }^{5}$. The approach can be better described into two levels. Level 1 illustrates a bird view to the subjectivity sentiment approach that we have used. In level 2 the data flows between the major components of the analysis are described (See Figure 5).

${ }^{5}$ https://www.djangoproject.com/ 


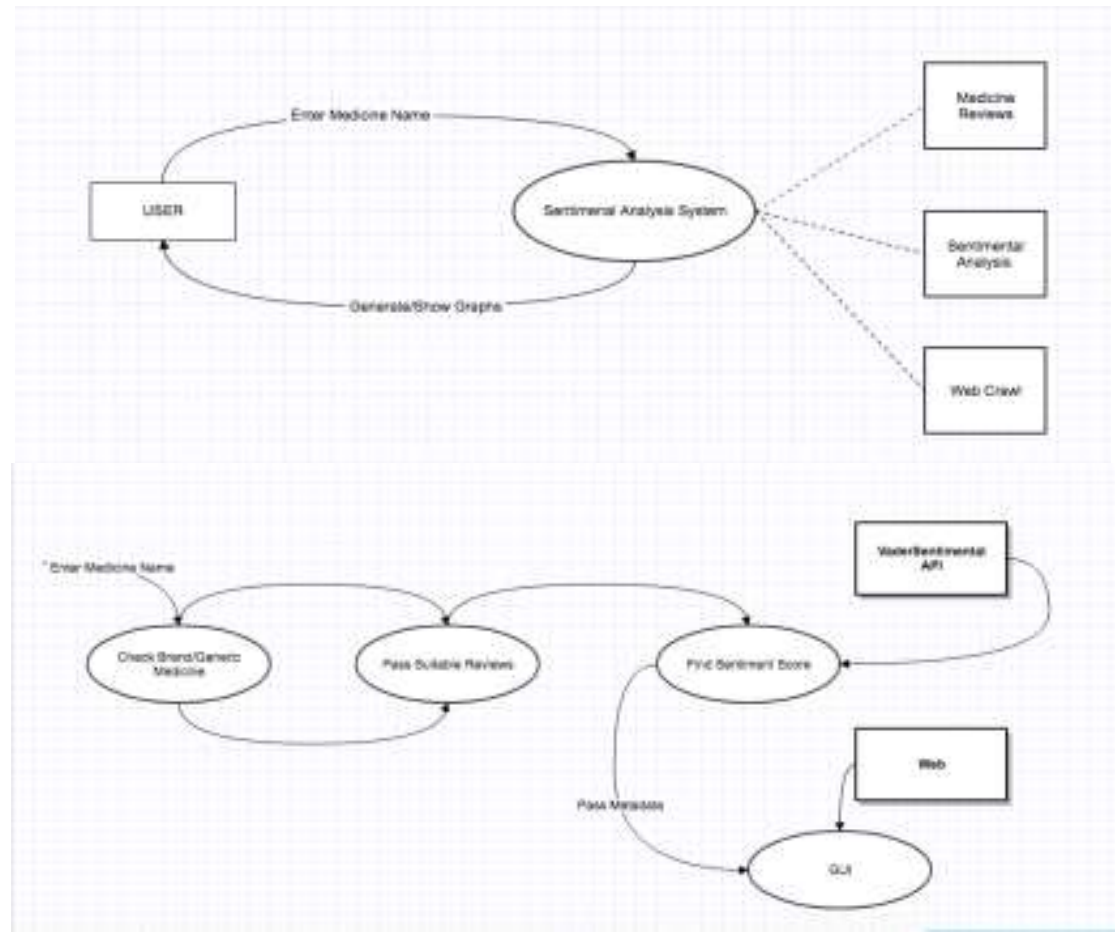

Fig. 5 Two levels description of the used sentiment approach.

Using our subjectivity sentiment framework we have collected 7976 reviews from the three mentioned drug reviews blogs. With our framework we are able to search for antihypertension drugs from these blogs and score the sentiments on them. Figure 6 illustrates our search for user's reviews on a drug like Norvasc between the periods of 2007-2016.

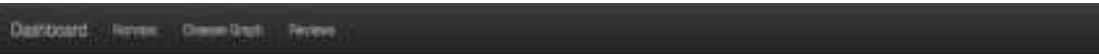 \\ Sentimental Analysis Result}
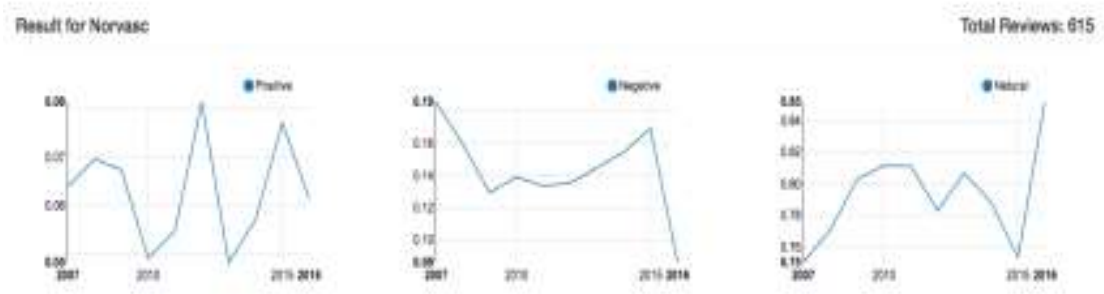

Fig. 6. Our Drug Subjectivity Dashboard

Based on the results obtained from these blogs we can compare different hypertension drugs. Figure 7 illustrates a comparison between Diovan and Mavic. 
Pesult for Diovan

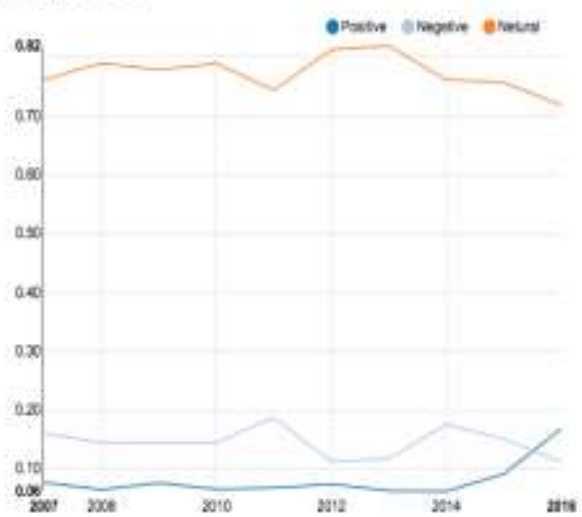

Fesult for Mavik

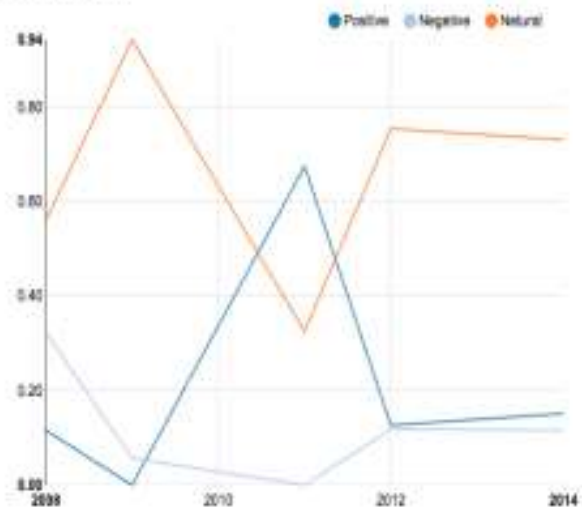

Fig. 7 One comparison between two antihypertension drugs

However, in most of the comparisons we have performed we found that the generic drugs have better sentiments compared to the brand drugs. This result may be somehow surprising and for this we decided to go and use the google trends ${ }^{6}$ for validation. In Figure 8 we provide our search for the twenty two antihypertension drugs that we have listed in our table 1. In this figure we are grouping each five drugs in one comparison and providing a final comparison for the winning drugs at the end. We also provide the map for the regions of the world where the drug have more sentiment.

\section{$\equiv$ GoogleTrends Compare}
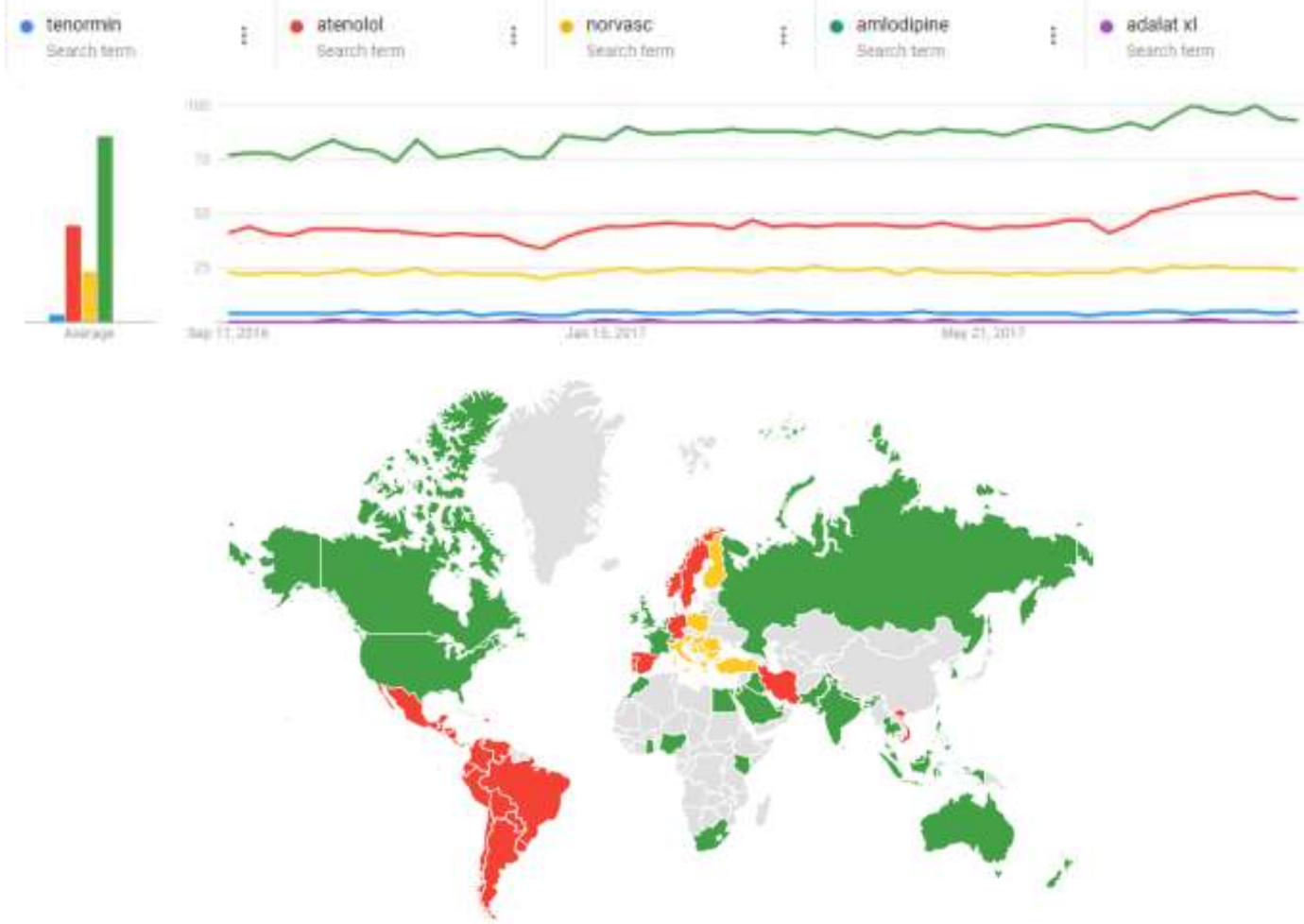

${ }^{6} \mathrm{https}: / /$ trends.google.com/trends/ 
International Journal of Advanced Science and Technology Vol.125 (2019)

\section{Google Trends Compare}

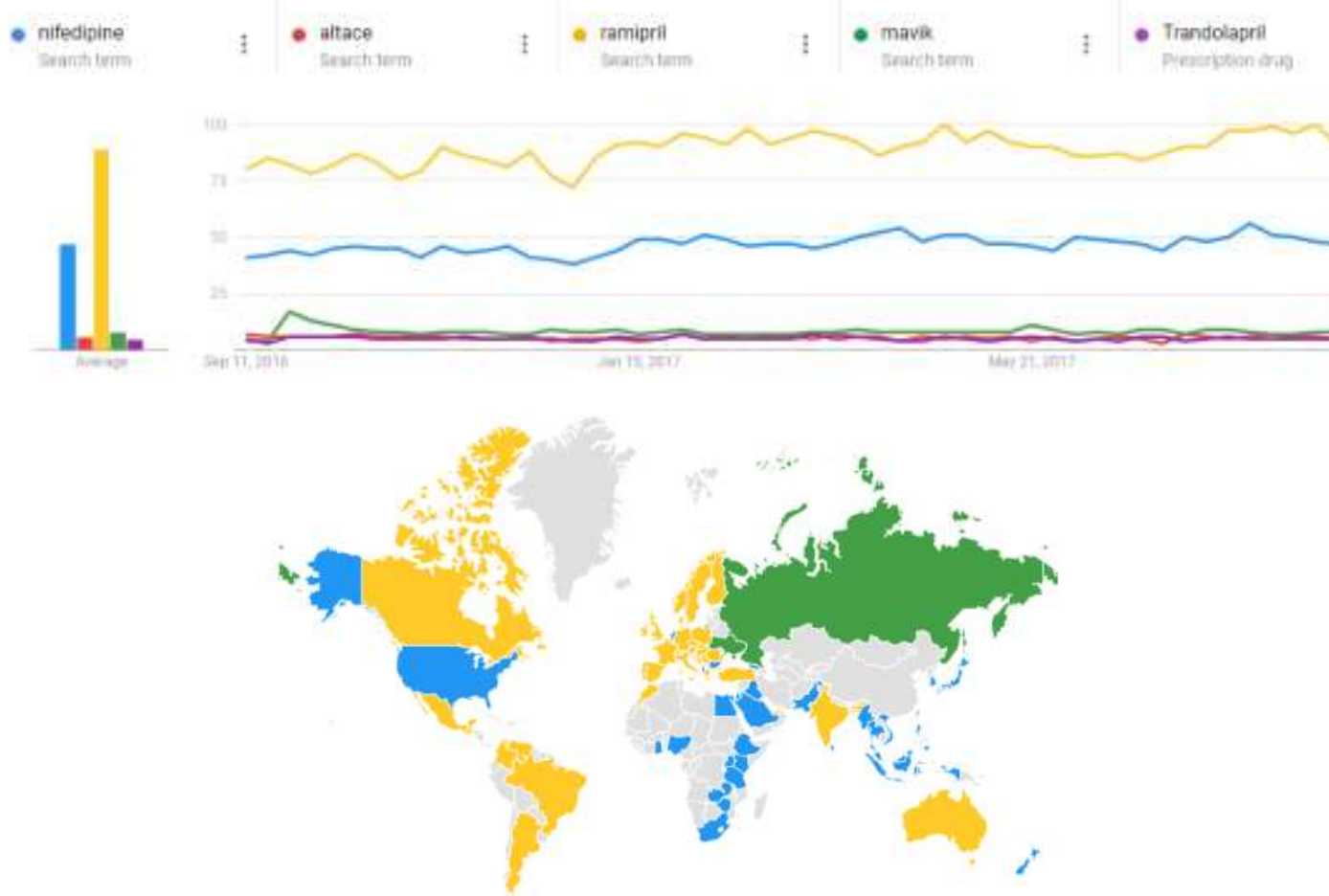

\section{Eoogle Trends Compare}

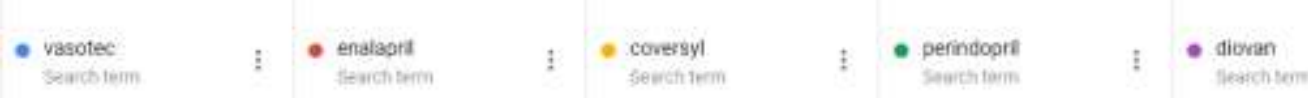
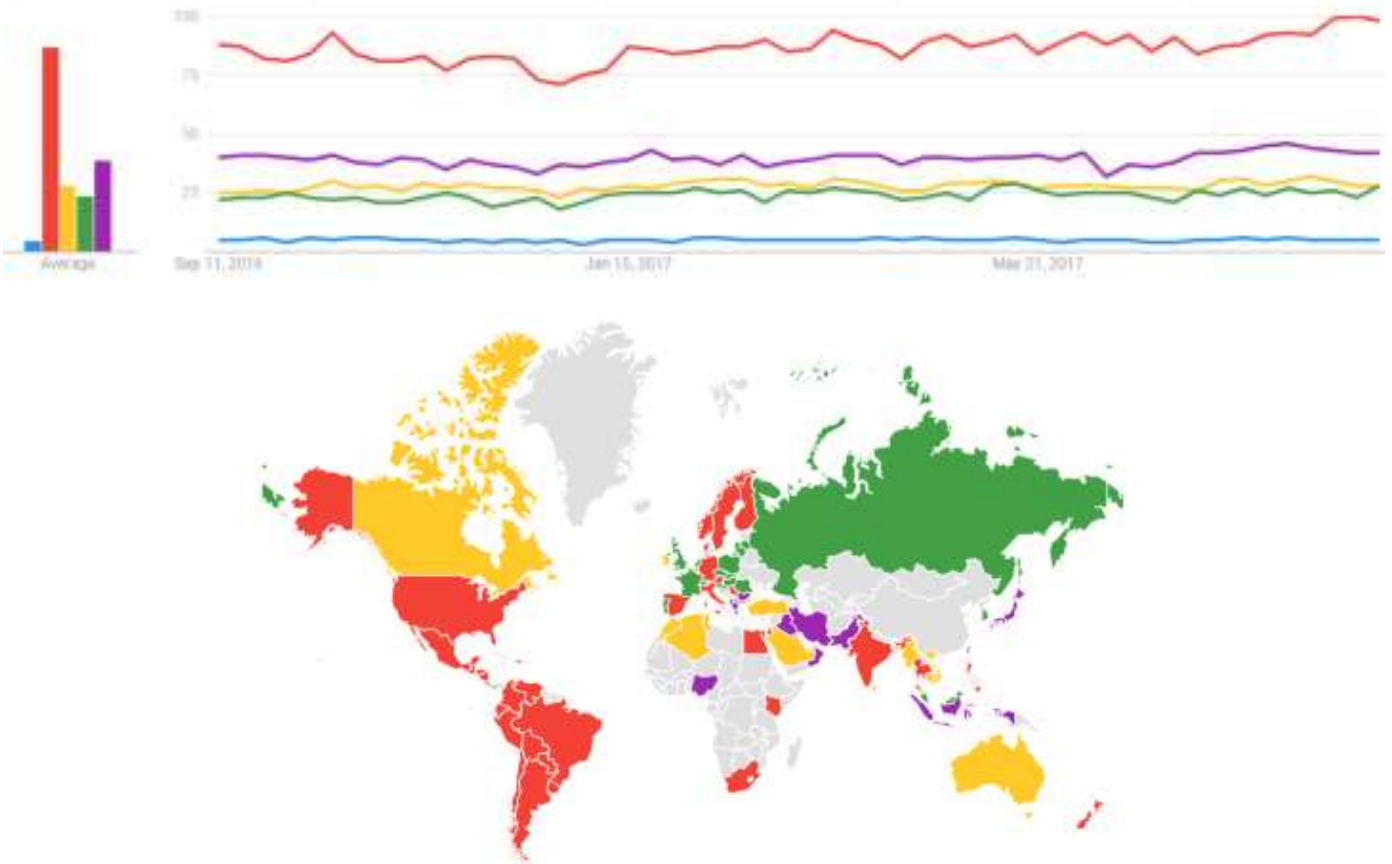


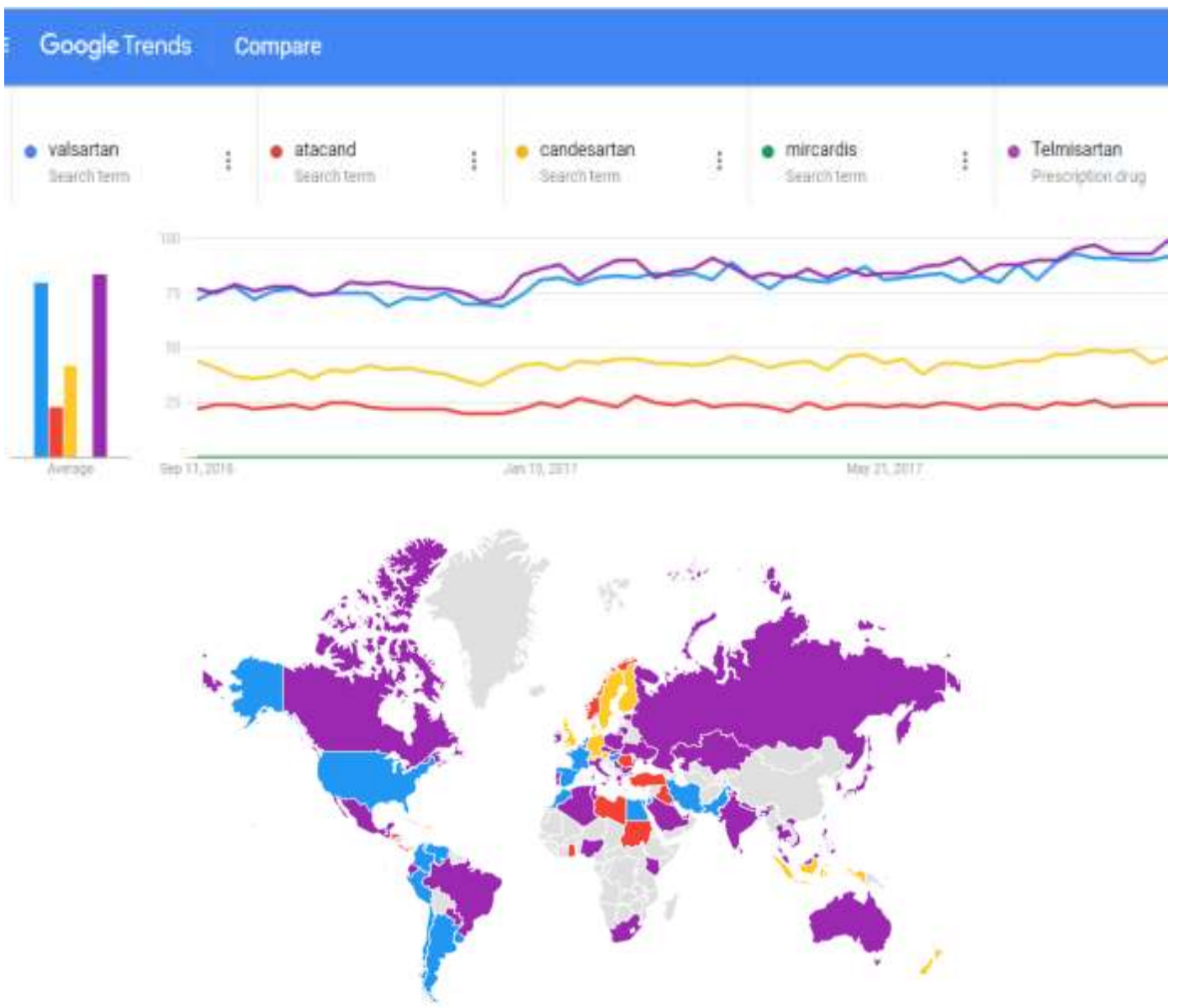

\section{Google Trendis: Compate}

- cozaar Sozart term

- Losartan Prescrition torag Add comparison
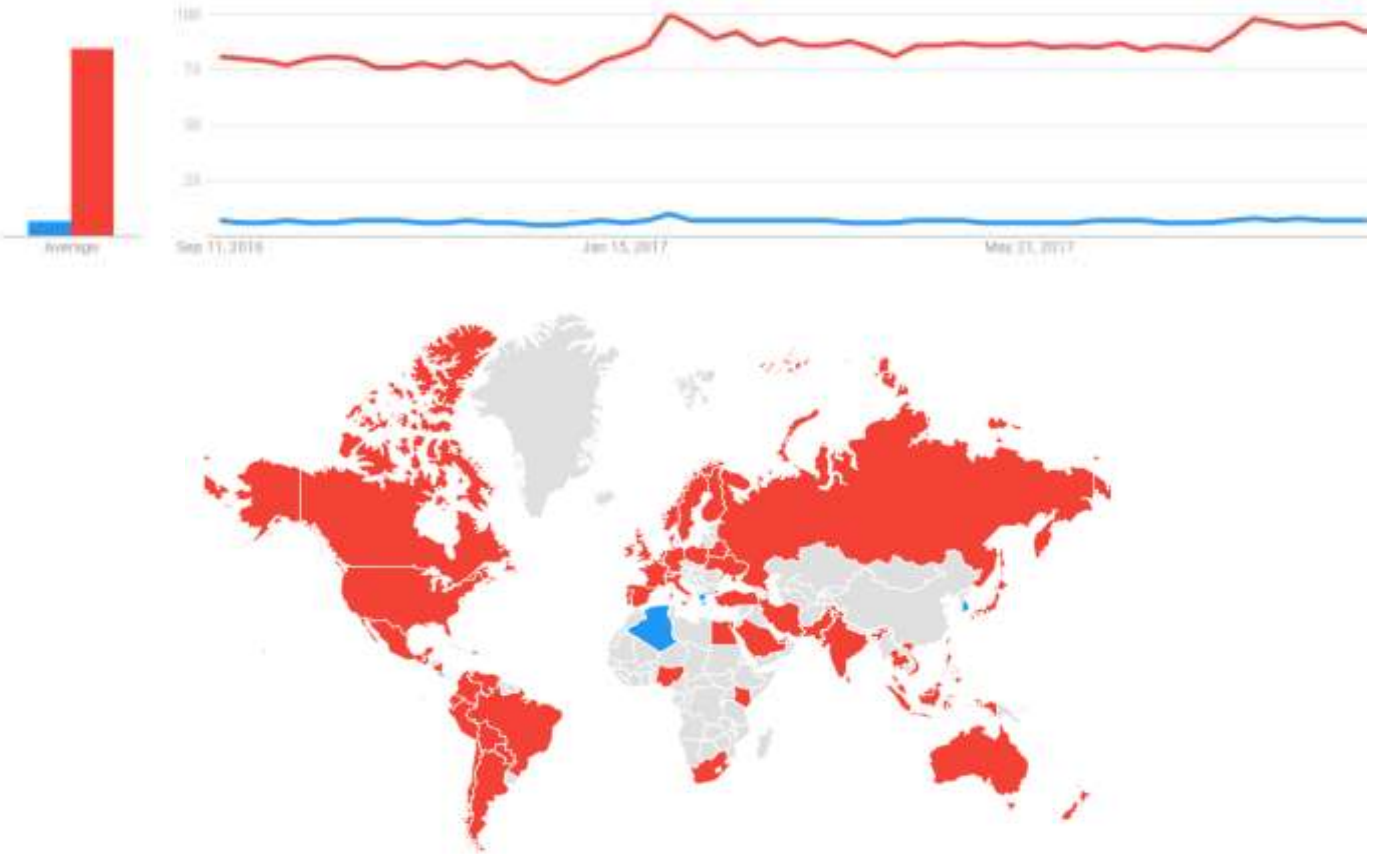


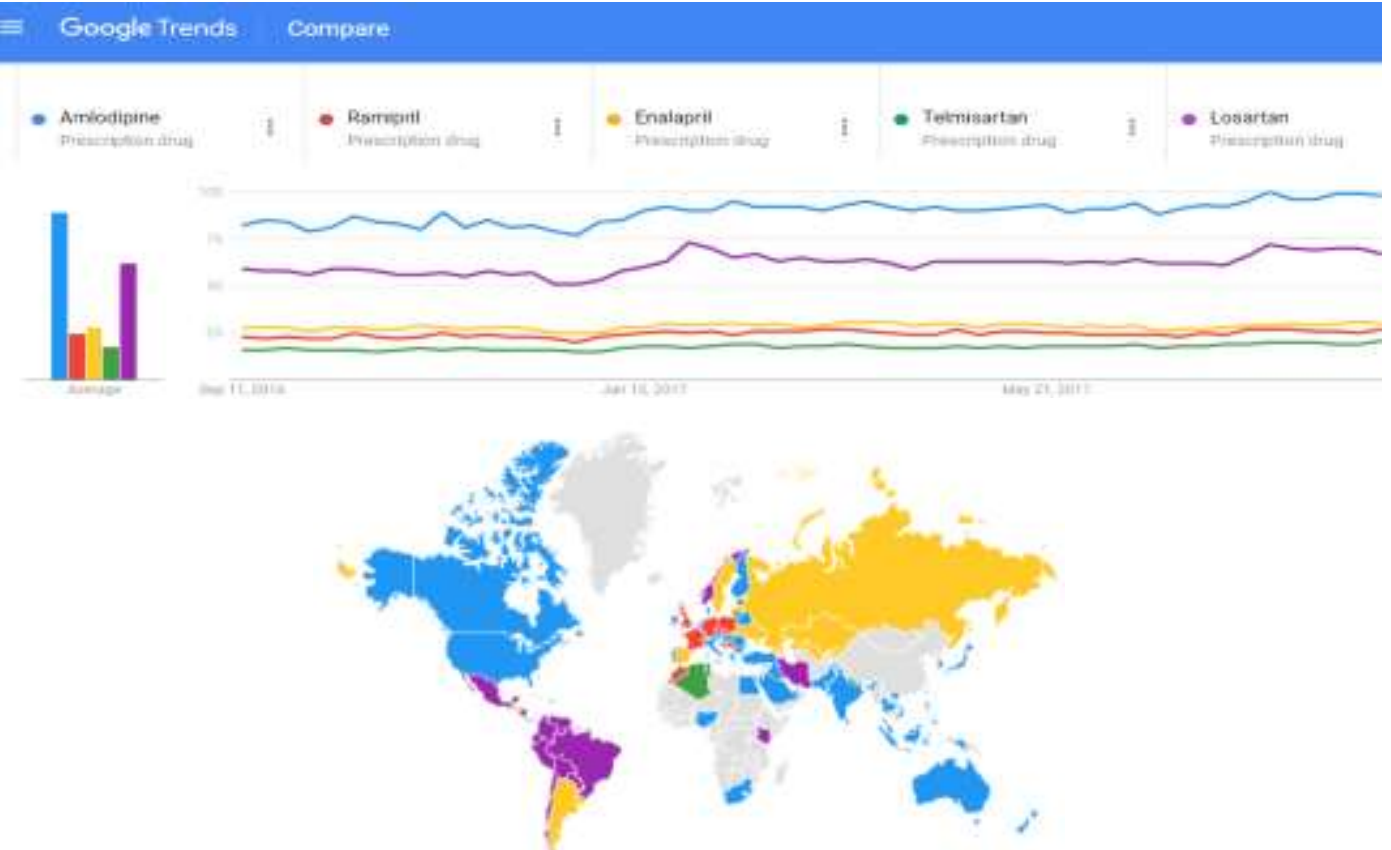

Fig. 8 Using Google Trends for Validating Our Findings

\section{CONCLUSIONS}

In this article we have described our initial phase of developing a framework for evaluating the sentiments on antihypertension drugs. The framework described avoids the complexities of using NLP and ML approaches. The sentiment approach uses a rule based approach as defined by the VADER API along with the average rating of the users for each drug as given by the notable drugs blogs like drugs.com. The framework employs an effective crawler based on the python scrapy API as well as using the python Django API for visualization. The crawled results shows more positive sentiments are with the Generic Antihypertension drugs compared with the Branded drugs. We are intending to expand our search and use other methods besides the Google trends to determine with greater confidence the difference between the sentiments when it comes to generic vs branded drugs.

\section{ACKNOWLEDGMENTS}

The first author would like to thank Professor Fiaidhi and Mohammed for their kind and detailed guideless as well as in supporting the awarded graduate teaching assistance ship. Moreover, the first author would like to thank Mr. Badal Shah the pharmacist at the Shoppers Drug Mart for his expert advice on antihypertension drugs.

\section{REFERENCES}

[1] Go AS, Mazaffarian D, Roger VL, et al., Heart disease and stroke statistics-2013 update: A report from the American Heart Association. Available on the internet at: http://www.circ.ahajournal.org/content/127/1/e6.

[2] Cooper-DeHoff, R. M., \& Elliott, W. J. (2013). Generic Drugs for Hypertension: Are They Really Equivalent? Current Hypertension Reports, 15(4), 340-345. http://doi.org/10.1007/s11906-013-0353-4

[3] Pang, B., Lee, L., Vaithyanathan, S.: Thumbs up? Sentiment classification using machine-learning techniques. In: The Conference on Empirical Methods in Natural Language Processing, pp. 79-86 (2002)

[4] Hutto, C.J. \& Gilbert, E.E. (2014). VADER: A Parsimonious Rule-based Model for Sentiment Analysis of Social Media Text. Eighth International Conference on Weblogs and Social Media (ICWSM-14). Ann Arbor, MI, June 2014. http://comp.social.gatech.edu/papers/icwsm14.vader.hutto.pdf 